https://helda.helsinki.fi

Observations on circumcenters in normed planes

Väisälä, Jussi

2017-09

Väisälä , J 2017 , ' Observations on circumcenters in normed planes ' , Beiträge zur Algebra und Geometrie , vol. 58 , no. 3 , pp. 607-615 . https://doi.org/10.1007/s13366-017-0338-6

http://hdl.handle.net/10138/307399

https://doi.org/10.1007/s13366-017-0338-6

acceptedVersion

Downloaded from Helda, University of Helsinki institutional repository.

This is an electronic reprint of the original article.

This reprint may differ from the original in pagination and typographic detail.

Please cite the original version. 


\title{
Observations on circumcenters in normed planes
}

\author{
Jussi Väisälä
}

\begin{abstract}
We give a necessary and sufficient condition for the existence of a circumcenter of a triangle in a normed plane. We also give alternative proofs of some results in [AMS2].

Keywords: circumcenter, bisector, normed plane.

2010 Mathematics Subject Classification: 52A21, 46B20
\end{abstract}

\section{Introduction}

1.1. Start. Throughout this note we let $E$ denote a normed (Minkowski) space with $\operatorname{dim} E=2$. A triangle in $E$ is a set $T=\{a, b, c\}$ of three noncollinear points. A point $x \in E$ is a circumcenter of $T$ if $\|x-a\|=\|x-b\|=\|x-c\|$. In a strictly convex space, a triangle has at most one circumcenter; see [MSW, Prop. 14.8], but a triangle need not always have a circumcenter; see [MSW, p. 128].

In Theorem 3.1 we characterize the triangles possessing a circumcenter. In Section 4 we give new proofs for some results of [AMS2] concerning acute triangles.

1.2. Notation. The open and closed discs with center $x$ and radius $r$ are written as $B(x, r)$ and $\bar{B}(x, r)$, and the boundary circle as $S(x, r)$. The center $x$ can be omitted if $x=0$. In particular, $\mathrm{S}(1)$ is the unit circle. For line segments in $E$ we use the customary notation $[a, b],[a, b),(a, b)$. Moreover, $\langle a, b\rangle$ is the line through $a$ and $b$, and $(a, b\rangle$ and $[a, b\rangle$ are the open and closed rays from $a$ through $b$. For real numbers $s, t$ we write $s \vee t=\max \{s, t\}, s \wedge t=\min \{s, t\}$.

\section{Bisectors}

2.1. Definitions and notation. Let $J=\left[b_{1}, b_{2}\right]$ be a line segment in $E$. The bisector of $J$ or of the pair $\left(b_{1}, b_{2}\right)$ is the set

$$
\text { bis } J=\operatorname{bis}\left[b_{1}, b_{2}\right]=\left\{x \in E:\left\|x-b_{1}\right\|=\left\|x-b_{2}\right\|\right\} .
$$

Let $L_{J}$ be one of the two supporting lines of the unit disc $\bar{B}(1)$ parallel to $J$, and let $u_{J}$ be the midpoint of the (possibly degenerate) line segment $L_{J} \cap S(1)$. Then the line

$$
l_{J}=\operatorname{span} u_{J}
$$

depends only on $J$ and not on the choice of $L_{J}$. We say that $l_{J}$ is the normal direction of $J$. Indeed, $l_{J}$ is one of the Birkhoff normals of $J$, written as $l_{J} \dashv L_{J}$; see [Th, 3.2.2] or [MSwa, 2.2]. Let

$$
Z_{J}=\left(b_{1}, b_{2}\right)+l_{J}
$$

be the open strip domain bounded by the parallel lines $b_{1}+l_{J}$ and $b_{2}+l_{J}$. 
2.2. Basic facts. We recall the basic facts about bisectors; see [MSwa, p. 121] and [JS].

Let $J=\left[b_{1}, b_{2}\right] \subset E$ be a line sgment. The point $a=\left(b_{1}+b_{2}\right) / 2$ belongs to bis $J$, and bis $J$ is symmetric with respect to $a$. If $L_{J} \cap S(1)$ is a singleton $\left\{u_{J}\right\}$, then bis $J$ is homeomorphic to a line and bis $J \subset Z_{J}$. Each line parallel to $J$ meets bis $J$ at a single point.

Assume that $L_{J} \cap S(1)$ is a nondegenerate line segment $\left[v_{1}, v_{2}\right]$, where $v_{2}-v_{1}$ has the same direction as $b_{2}-b_{1}$. Now the rays $\left[b_{1}, b_{1}+v_{2}\right\rangle$ and $\left[b_{2}, b_{2}+v_{1}\right\rangle$ meet at a point $y_{J} \in a+l_{J}$. Each line parallel to $J$ between $y_{J}$ and $J$ meets bis $J$ at a single point, which belongs to the solid triangle conv $\left\{b_{1}, b_{2}, y_{J}\right\}$. Thus bis $J$ contains an arc $\gamma$ with endpoints $y_{J}$ and $y_{J}^{*}=2 a-y_{J}$. In addition, bis $J$ contains two closed convex cones $V$ and $V^{*}=2 a-V$ where $\partial V$ consists of the rays $\left[b_{i}, y_{J}\right\rangle \backslash\left[b_{i}, y_{J}\right), i=1,2$. It follows that bis $J$ contains the reduced bisector

$$
\operatorname{bis}_{0} J=\operatorname{bis}_{0}\left[b_{1}, b_{2}\right]=\gamma \cup\left(\left(a+l_{J}\right) \backslash\left(y_{J}^{*}, y_{J}\right)\right) .
$$

Each line parallel to $J$ meets $\operatorname{bis}_{0} J$ at a single point, and $\operatorname{bis}_{0} J \subset Z_{J}$. If $L_{J} \cap S(1)=\left\{u_{J}\right\}$, we write $\operatorname{bis}_{0} J=\operatorname{bis} J$.

Observe that the bisector bis $J$ is always connected.

2.3. Lemma. If the normal directions of the segments $J$ and $J^{\prime}$ are different, then $\operatorname{bis}_{0} J \cap \operatorname{bis}_{0} J^{\prime} \neq \varnothing$.

Proof. The set $Q=Z_{J} \cap Z_{J^{\prime}}$ is a parallelogram. There is an $\operatorname{arc} \alpha \subset \operatorname{bis}_{0} J$ joining a pair of opposite sides of $Q$ in $\bar{Q}$, and another $\operatorname{arc} \alpha^{\prime} \subset$ bis $_{0} J^{\prime}$ joining the other pair. Now $\alpha \cap \alpha^{\prime} \neq \varnothing$ (see e.g. [Ne, V.11.8]), and the lemma follows.

The following lemma is trivial:

2.4. Lemma. (1) The line $l_{J}$ is never parallel to $J$.

(2) Suppose that $J$ and $J^{\prime}$ are line segments in $E$ and that $S(1)$ contains a line segment parallel to $J$. If $l_{J}=l_{J^{\prime}}$, then $J$ and $J^{\prime}$ are parallel.

\section{Circumcenters}

By a triangle we mean a set $T=\{a, b, c\}$ of three noncollinear points in $E$. A point $p$ is a circumcenter of $T$ if $\|p-a\|=\|p-b\|=\|p-c\|$. Equivalently, $p \in$ bis $[x, y]$ for each pair $x, y \in T$. Clearly it suffices that this holds for two bisectors.

We now give our main result.

3.1. Theorem. A triangle $T \subset E$ possesses no circumcenter if and only if the normal directions of its sides are all equal.

Proof. Let $T=\left\{t_{1}, t_{2}, t_{3}\right\}$ and let $J_{i}$ be the side opposite to $t_{i}$. If two of the lines $l_{i}=l_{J_{i}}$ are different, then $T$ has a circumcenter by Lemma 2.3 .

Conversely, assume that $l_{1}=l_{2}=l_{3}=: l$. By Lemma 2.4, no side of $T$ is parallel to $l$ or to a segment in $S(1)$. Hence bis $J_{i}$ is contained in the strip $Z_{i}=Z_{J_{i}}$, which is bounded by the parallel lines $t_{j}+l$ and $t_{k}+l$. It follows that two of these strips, say $Z_{1}$ and $Z_{2}$, are disjoint and contained in the third strip $Z_{3}$. Thus bis $J_{1} \cap$ bis $J_{2}=\varnothing$, and the theorem follows.

A point $x \in S(1)$ is a smooth point if $S(1)$ has a tangent at $x$. If $x$ is a smooth point, then also $-x$ is a smooth point. Hence it makes sense to say that $u_{J}$ is a smooth point, 
although $u_{J}$ is determined by $J$ only up to sign. If $l$ is a line through the origin and if the points in $l \cap S(1)$ are smooth points, we say that $S(1)$ is smooth in the direction $l$.

Suppose that $T \subset E$ is a triangle and that $T$ has a side $J$ such that $u_{J}$ is a smooth point of $S(1)$. If $J^{\prime}$ is another side of $T$, then $l_{J^{\prime}} \neq l_{J}$. By Theorem 3.1 this implies:

3.2. Theorem. Suppose that the unit circle is smooth in the normal direction of a side of a triangle $T$ (equivalently, $T$ has a side parallel to a tangent of the unit circle). Then $T$ has a circumcenter.

As a corollary we obtain the classical result:

3.3. Theorem. If $E$ is smooth, then every triangle has a circumcenter.

3.4. Remark. Theorem 3.3 was proved by Mayer [Ma, p. 518] in 1935 (for strictly convex spaces) with a fairly long proof. Kramer and Németh [KN] gave an elegant proof based on Brouwer's fixpoint theorem; see [MSW, p. 32].

\section{Acute triangles}

In this section we give an alternative treatment of some results of J. Alonso, H. Martini and M. Spirova [AMS2] concerning acute triangles. Let $T=\left\{t_{1}, t_{2}, t_{3}\right\}$ be a triangle in $E$, and let $m_{i j}$ denote the midpoint $\left(t_{i}+t_{j}\right) / 2$ of the side $\left[t_{i}, t_{j}\right]$. The triangle $T$ is called acute (in [AMS2] norm-acute) if $\left\|t_{k}-m_{i j}\right\|>\left\|t_{i}-m_{i j}\right\|=\left\|t_{i}-t_{j}\right\| / 2$ for $\{i, j, k\}=\{1,2,3\}$. In a Euclidean plane this means that all angles of $T$ are less than $\pi / 2$.

The following result was proved in [AMS2, Th. 6.1]:

\subsection{Theorem. Every acute triangle $T=\left\{t_{1}, t_{2}, t_{3}\right\} \subset E$ has a circumcenter.}

We shall give three new proofs for this result. The first proof is short and makes use of bisectors, the second proof is an application of Theorem 3.1, and the third proof is a by-product of the proof of Theorem 4.6 and makes use of minimal enclosing discs.

First proof. As in the proof of Theorem 3.1, we let $J_{i}$ denote the side of $T$ opposite to $t_{i}$. Assume that $J_{3}=\left[t_{1}, t_{2}\right]$ is the longest side of $T$. Set $s=\left\|t_{1}-m_{12}\right\|=\left\|t_{1}-t_{2}\right\| / 2$. There is a point $y \in J_{1} \cup J_{2}$ with $\left\|y-t_{1}\right\|=\left\|y-t_{2}\right\| \geq s$. We may assume that $y \in J_{1}$. Define $f:$ bis $J_{3} \rightarrow \mathrm{R}$ by

$$
f(x)=\left\|x-t_{3}\right\|-\left\|x-t_{1}\right\| .
$$

By acuteness we have $f\left(m_{12}\right)>0$. As $\left\|t_{3}-t_{2}\right\| \leq\left\|t_{1}-t_{2}\right\|=2 s$ and $\left\|y-t_{3}\right\|=$ $\left\|t_{3}-t_{2}\right\|-\left\|y-t_{2}\right\|$, we obtain

$$
f(y)=\left\|t_{3}-t_{2}\right\|-\left(\left\|y-t_{2}\right\|+\left\|y-t_{1}\right\|\right) \leq 2 s-2 s=0 .
$$

As bis $J_{3}$ is connected and $f$ is continuous, there is a point $x_{0} \in J_{3}$ with $f\left(x_{0}\right)=0$, and this $x_{0}$ is a circumcenter of $T$.

Second proof. Write $J_{i}=\left[t_{j}, t_{k}\right]$ as above and set $l_{i}=l_{J_{i}}$; see 2.1. Assume that $l_{1}=l_{2}=l_{3}$. By Theorem 3.1 it suffices to show that this leads to a contradiction.

Assume again that $J_{3}=\left[t_{1}, t_{2}\right]$ is the longest side of $T$. We may assume that $\left\|t_{1}\right\|=$ $\left\|t_{2}\right\|=1$ and that $t_{1}+t_{2}=0$. Let $L_{3}=\left\langle t_{1}, t_{2}\right\rangle$ be the line containing $J_{3}$ and let $H$ be the open half plane with $\partial H=L_{3}$ and $t_{3} \in H$. Let $u_{3}=u_{J_{3}}$ be as in 2.1. Thus 
$u_{3} \in S(1) \cap H$ and $u_{3}+L_{3}$ is a supporting line of $\bar{B}(1)$, that is, $\left(u_{3}+L_{3}\right) \cap B(1)=\varnothing$. Then $\left(u_{3}+L_{3}\right) \cap \bar{B}(1)$ is either $\left\{u_{3}\right\}$ or a line segment with midpoint $u_{3}$. For $i=1,2$ write $M_{i}=\left\langle t_{i}, u_{3}\right\rangle$ and let $H_{i}$ be the open half plane such that $\partial H_{i}=M_{i}$ and $0 \notin H_{i}$.

As $\Delta:=\operatorname{conv}\left\{t_{1}, t_{2}, u_{3}\right\} \subset \bar{B}(1)$ and $T$ is acute, we have $t_{3} \in H \backslash \Delta=H \cap\left(H_{1} \cup H_{2}\right)$. We may assume that $t_{3} \in H \cap H_{2}$. The ray $R=\left[t_{1}, u_{3}\right\rangle+2 t_{2}$ divides $H_{2} \cap H$ into two angular domains $D_{1}=H_{2} \cap\left(H_{1}+2 t_{2}\right)$ and $D_{2}=H_{2} \backslash\left(\bar{H}_{1}+2 t_{2}\right)$.

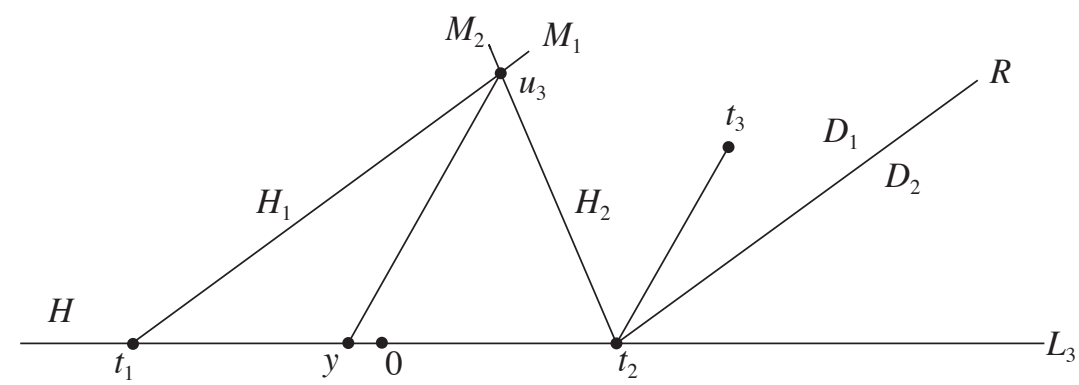

Fig. 1. Second proof, Case 1

Case 1. $t_{3} \in \bar{D}_{1}$. Now there is $y \in J_{3}$ such that $\left[y, u_{3}\right]$ is parallel to $J_{1}=\left[t_{2}, t_{3}\right]$. As $l_{1}=l_{3}$, we have $u_{1}=u_{3}$, whence $\left\langle u_{3}, y\right\rangle$ is a supporting line of $B(1)$. Hence $y=t_{1}$ and $\left[t_{1}, u_{3}\right] \subset S(1)$. By Lemma 2.4(2) this implies that $J_{1}$ and $J_{3}$ are parallel, a contradiction.

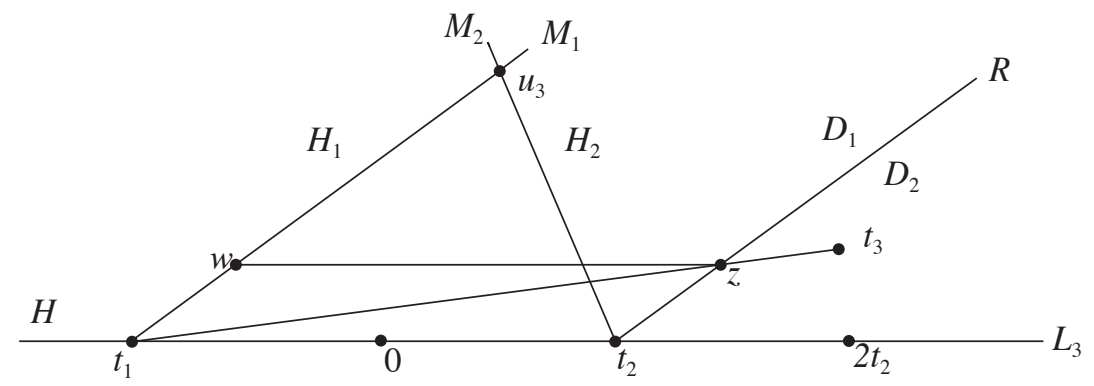

Fig. 2. Second proof, Case 2

Case 2. $t_{3} \in D_{2}$. We prove that $J_{3}$ cannot be the longest side of $T$. There is a point $z \in\left[t_{1}, t_{3}\right) \cap R$. As $\left\|t_{1}-t_{3}\right\| \leq\left\|t_{1}-t_{2}\right\|=2$, it suffices to show that $\left\|z-t_{1}\right\| \geq 2$. By convexity of the disc $\bar{B}\left(t_{1}, 2\right)$, it suffices to prove this for $z$ close to $t_{2}$. We prove it in the case where $\left\|z-t_{2}\right\| \leq\left\|t_{1}-u_{3}\right\|$. Then we can write $z=w+2 t_{2}$ where $w \in\left[t_{1}, u_{3}\right]$. As $\left\|2 t_{2}-z\right\|=\|w\| \leq 1$, we obtain

$$
3=\left\|2 t_{2}-t_{1}\right\| \leq\left\|2 t_{2}-z\right\|+\left\|z-t_{1}\right\| \leq 1+\left\|z-t_{1}\right\|,
$$

and thus $\left\|z-t_{1}\right\| \geq 2$.

4.2. Minimal enclosing discs. Let $T \subset E$ be a triangle and set

$$
r_{0}=\inf \{r: T \subset \bar{B}(x, r) \text { for some } x \in E\} .
$$

A closed disc $\bar{B}\left(x_{0}, r_{0}\right)$ is a minimal enclosing disc of $T$ if $T \subset \bar{B}\left(x_{0}, r_{0}\right)$. The number $r_{0}$ is the minimal enclosing radius of $T$. An easy compactness argument shows that every triangle has at least one minimal enclosing disc. Minimal enclosing discs of triangles in normed planes were extensively studied in [AMS2]. We shall give an alternative treatment of [AMS2, Th. 6.5] on minimal enclosing discs of acute triangles with precisely one circumcenter. We give a somewhat different formulation, which also gives a third proof 
for Theorem 4.1. We use the notation $\operatorname{MEDC}(T)$ of [AMS2] for the locus of the centers of all minimal enclosing discs of $T$.

The following lemma is contained in the proof of $[\mathrm{AMS} 2,6.5]$. We recall the proof.

4.3. Lemma. If $\bar{B}(x, r)$ is a minimal enclosing disc of a triangle $T=\left\{t_{1}, t_{2}, t_{3}\right\} \subset E$, then $S(x, r)$ contains at least two vertices of $T$.

Proof. Clearly $S(x, r)$ contains at least one point $t_{1} \in T$. Assume that $t_{2}, t_{3} \in B(x, r)$. Setting $\delta=r-\left(\left\|t_{2}-x\right\| \vee\left\|t_{3}-x\right\|\right)$ we have $0<\delta<r$. Let $y \in\left[t_{1}, x\right]$ be the point with $\|y-x\|=\delta / 2$. For $i=1,2$ we have

$$
\left\|t_{i}-y\right\| \leq\left\|t_{i}-x\right\|+\|x-y\| \leq r-\delta+\delta / 2=r-\delta / 2 .
$$

As $\left\|t_{1}-y\right\|=r-\delta / 2$, we have $T \subset \bar{B}(y, r-\delta / 2)$, and $r$ is not minimal.

4.4. Notation. Given a circle $S=S(p, r) \subset E$ and a point $a \in S$, we let

$$
a^{*}=2 p-a
$$

denote the point of $S$ opposite to $a$. In particular, if $p=0$, then $a^{*}=-a$.

4.5. Lemma. Let $a, b \in S=S(p, r) \subset E$ and $z=(a+b) / 2$. Then the following conditions are equivalent:

(1) $\|a-b\|=2 r$.

(2) $\left[a, b^{*}\right] \subset S$,

(3) $[z, p] \subset S(a, r) \cap S(b, r)$.

Proof. If $z=p$, then $a=b^{*}$, and the lemma holds in the degenerate form $\left[a, b^{*}\right]=$ $\{a\},[z, p]=\{p\}$. Assume that $z \neq p$. We show that $(1) \Rightarrow(3) \Rightarrow(2) \Rightarrow(1)$.

$(1) \Rightarrow(3)$ : Let $x \in[z, p]$. As $\|p-a\|=\|z-a\|=r$, we have $\|x-a\| \leq r$ and similarly $\|x-b\| \leq r$. Hence

$$
2 r=\|a-b\| \leq\|a-x\|+\|x-b\| \leq 2 r
$$

and (3) follows.

$(3) \Rightarrow(2)$ : Let $x \in\left[a, b^{*}\right]$ and set $y=\left(a+b^{*}\right) / 2$. The translation $f(x)=x+y-z$ maps $b, z, p$ to $p, y, b^{*}$. Hence $\left[y, b^{*}\right]=f[z, p] \subset f S(b, r)=S(p, r)$.

$(2) \Rightarrow(1)$ : Define $g: E \rightarrow E$ by $g(x)=2 x-b^{*}$ and let $y$ be as above. Then $g(y)=$ $a, g(p)=b$, whence $\|a-b\|=2\|y-p\|=2 r$.

We next prove a version of Theorem 6.5 of [AMS2]. We do not assume that Theorem 4.1 is known but get a new proof of it.

4.6. Theorem. Suppose that $T=\left\{t_{1}, t_{2}, t_{3}\right\} \subset E$ is an acute triangle with $\left\|t_{2}-t_{3}\right\| \leq$ $\left\|t_{1}-t_{3}\right\| \leq\left\|t_{1}-t_{2}\right\|$ and that $T$ has at most one circumcenter. Suppose also that $\bar{B}=$ $\bar{B}\left(x_{0}, r\right)$ is a minimal enclosing disc of $T$. There are three possibilities:

(1) $\left\|t_{i}-t_{j}\right\|<2 r$ for all $i, j$,

(2) $\left\|t_{1}-t_{3}\right\|<\left\|t_{1}-t_{2}\right\|=2 r$,

(3) $\left\|t_{1}-t_{3}\right\|=\left\|t_{1}-t_{2}\right\|=2 r$.

In cases (1) and (3) we have $T \subset S=S\left(x_{0}, r\right)$ and thus $x_{0}$ is the unique circumcenter of $T$. Moreover, $\bar{B}$ is the only minimal enclosing disc of $T$.

In case (2), $T$ has the unique circumcenter $y \in\left[m_{12}, x_{0}\right]$ and $T \subset S(y, r)$ where $m_{12}=\left(t_{1}+t_{2}\right) / 2$. Moreover, the set $A=S\left(t_{1}, r\right) \cap S\left(t_{2}, r\right)$ is a line segment $[u, v] \supset$ $\left[m_{12}, x_{0}\right]$ aligned as $u, m_{12}, x_{0}, v$, and $\operatorname{MEDC}(T)=[y, v]$. 
Proof. (1): Let $\{i, j, k\}=\{1,2,3\}$. By Lemma 4.3 we may assume that $t_{i}, t_{j} \in S$. Assume that $\left\|t_{k}-x_{0}\right\|<r$. As $\left\|t_{i}-t_{j}\right\|<2 r$, we have $\left[m_{i j}, x_{0}\right) \subset B\left(t_{1}, r\right) \cap B\left(t_{2}, r\right)$ where $m_{i j}=\left(t_{i}+t_{j}\right) / 2$. Set

$$
\delta=\left(r-\left\|t_{k}-x_{0}\right\|\right) \wedge\left\|m_{i j}-x_{0}\right\|
$$

and let $z \in\left[m_{i j}, x_{0}\right]$ be the point with $\left\|z-x_{0}\right\|=\delta / 2$. Then

$$
\left\|t_{k}-z\right\| \leq\left\|t_{k}-x_{0}\right\|+\left\|x_{0}-z\right\| \leq r-\delta+\delta / 2=r-\delta / 2 .
$$

Hence $T \subset B(z, r)$ and $\bar{B}$ cannot be a minimal enclosing disc of $T$. Thus $T \subset S$. As the argument is valid for any minimal enclosing disc of $T, \bar{B}$ is unique.

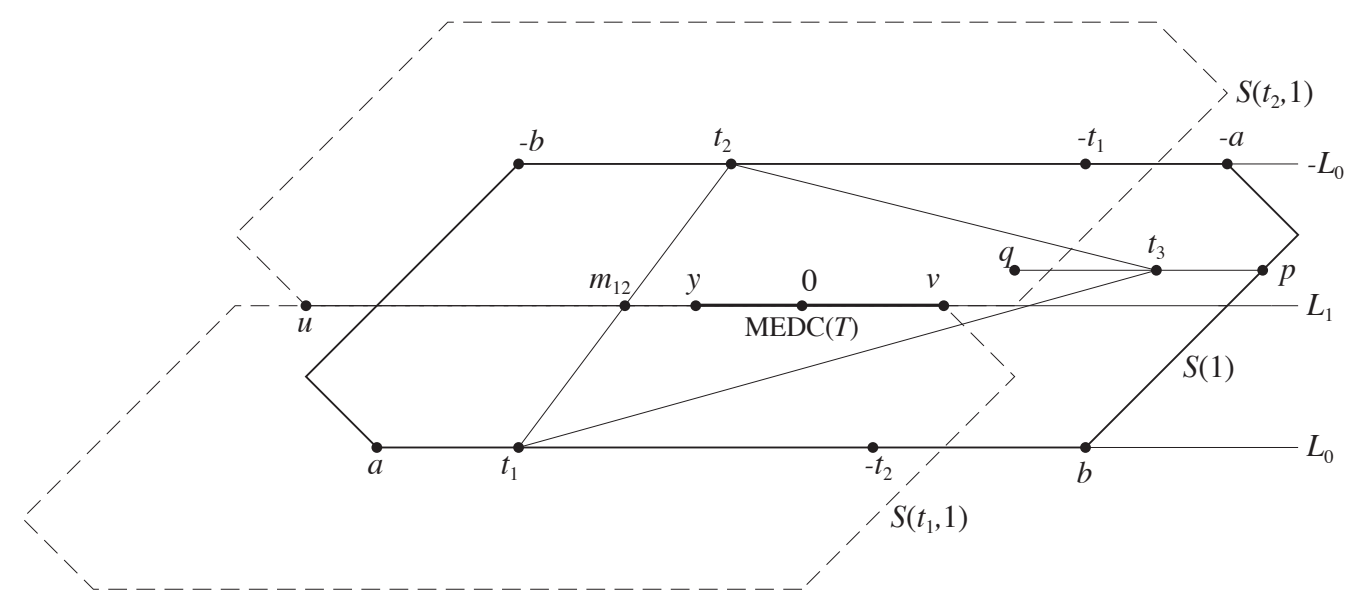

Fig. 3. Th. 4.6, Case (2)

(2) and (3): We normalize $x_{0}=0, r=1$. By acuteness we have $\left\|t_{3}-m_{12}\right\|>$ $\left\|t_{1}-m_{12}\right\|=1$, whence $m_{12} \neq 0$. By Lemma 4.5 we have $\left[t_{1},-t_{2}\right] \subset S(1)$. Setting $L_{0}=\left\langle t_{1},-t_{2}\right\rangle$ and $J_{0}=[a, b]=L_{0} \cap S(1)$ we have $\left[t_{1},-t_{2}\right] \subset J_{0}$ and $\left[t_{2},-t_{1}\right] \subset-J_{0}$, and the parallelogram with vertices $a, b,-a,-b$ lies in $\bar{B}(1)$. Set $\alpha=\|a-b\|$. Then each line parallel to $L_{0}$ between $L_{0}$ and $-L_{0}$ meets $\bar{B}(1)$ in a segment of length $\geq \alpha$.

By Lemma 4.5 , the set $A$ is a line segment $[u, v]$ containing $\left[m_{12}, 0\right]$. Set $L_{1}=\left\langle m_{12}, 0\right\rangle$. As the lengths of the segments $S\left(t_{i}, 1\right) \cap L_{1}, i=1,2$, are $\alpha$, we have $\|u-v\| \leq \alpha$. As $\left\|t_{3}\right\| \leq 1<\left\|t_{3}-m_{12}\right\|$, there is a point $y \in\left(m_{12}, 0\right]$ with $\left\|t_{3}-y\right\|=1$. Then $T \subset S(y, 1)$ and $y$ is the unique circumcenter of $T$. By convexity of the disc $\bar{B}\left(t_{3}, 1\right)$ we have $\left\|x-t_{3}\right\|>1$ for all $x$ in the open ray $\left(y, m_{12}\right\rangle$.

In case (3) the argument above is valid with $t_{2}$ replaced by $t_{3}$. Hence the unique circumcenter of $T$ lies in $\left[m_{12}, 0\right] \cap\left[m_{13}, 0\right]=\{0\}$, and case (3) is proved.

In case (2) we have $\operatorname{MEDC}(T)=[u, v] \cap \bar{B}\left(t_{3}, 1\right)$. Since $[u, y) \cap \bar{B}\left(t_{3}, 1\right)=\varnothing$ and $\left\|y-t_{3}\right\|=1$, it remains to show that $\left\|v-t_{3}\right\| \leq 1$.

Set $p=t_{3}-y, q=t_{3}-v$. Then $\langle q, p\rangle$ is parallel to $L_{0}, p \in S(1)$, and

$$
\|q-p\|=\|v\|+\|y\|=\|y-v\| \leq\|u-v\| \leq \alpha,
$$

which implies that $q \in \bar{B}(1)$. 
4.7. Remarks. 1. As a by-product, the proof of 4.6 gives: If $T$ is acute and if $T$ is contained in a unique circle $S(x, r)$, then $r$ is the minimal enclosing radius of $T$. This is a special case of [AMS2, Th. 6.4].

2. As another by-product, we get a third new proof for the existence of a circumcenter of acute triangles (Theorem 4.1, [AMS2, Th. 6.4]). Indeed, suppose that $T$ is an acute triangle without a circumcenter and choose a minimal enclosing disc $\bar{B}\left(x_{0}, r\right)$ of $T$. Then, in cases (1) and (3), $x_{0}$ is a circumcenter and in case (2), $y$ is a circumcenter.

4.8. We recall Theorem 6.5 of [AMS2] and show how it follows from 4.6.

Theorem 6.5 of $[\mathrm{AMS} 2]$. Let $T=\left\{t_{1}, t_{2}, t_{3}\right\}$ be an acute triangle, and assume that $S(1)$ is the unique circle containing $T$. There are two possibilities:

(i) There is no pair $(i, j)$ such that $\left[t_{i},-t_{j}\right]$ lies in the relative interior of a line segment in $S(1)$. Then $\bar{B}(1)$ is the unique minimal enclosing disc of $T$.

(ii) $\left[t_{1},-t_{2}\right]$ is in the relative interior of a segment $\left[t_{1}^{\prime},-t_{2}^{\prime}\right]$ of $S(1)$, that is assumed to be maximal, aligned as $t_{1}^{\prime}, t_{1},-t_{2},-t_{2}^{\prime}$. Then $\operatorname{MEDC}(T)=\left[0, t_{1}-t_{1}^{\prime}\right] \cap\left[0, t_{2}-t_{2}^{\prime}\right]$.

Proof. From Remark 4.7.1 it follows that the circumradius of $T$ is 1 . Hence we may apply Theorem 4.6 with $x_{0}=0, r=1$.

We prove (i) and (ii) without the words "the relative interior of". Call the new versions (i') and (ii'). Then, if $\left[t_{1},-t_{2}\right]$ is contained in $S(1)$ but not in the relative interior of any other segment in $S(1)$, we have $t_{1}=t_{1}^{\prime}$ or $t_{2}=t_{2}^{\prime}$, and (ii') gives $\operatorname{MEDC}(T)=$ $[0,0]=\{0\}$ as in (i).

Case (i'): By Lemma 4.5 we have $\left\|t_{i}-t_{j}\right\|<2$ for all $i, j$. Now 4.6(1) implies that $\bar{B}(1)$ is the only minimal enclosing disc of $T$.

Case (ii'): By Lemma 4.5 we have $\left\|t_{1}-t_{2}\right\|=2$. Hence we have the situation of $4.6(2)$ or 4.6(3). We use the notation of the proof of 4.6(2) but observe that the normalization there is different, and the point $y$ is now the origin. We have now $J_{0}=\left[t_{1}^{\prime},-t_{2}^{\prime}\right],-J_{0}=$ $\left[t_{2}^{\prime},-t_{1}^{\prime}\right]$, and

$$
\begin{aligned}
& S\left(t_{1}, 1\right) \cap L_{1}=-J_{0}+t_{1}=\left[t_{1}+t_{2}^{\prime}, t_{1}-t_{1}^{\prime}\right], \\
& S\left(t_{2}, 1\right) \cap L_{1}=J_{0}+t_{2}=\left[t_{2}+t_{1}^{\prime}, t_{2}-t_{2}^{\prime}\right] .
\end{aligned}
$$

The intersection of these segments is $[u, v]$, whence $\operatorname{MEDC}(T)=[0, v]=\left[0, t_{1}-t_{1}^{\prime}\right] \cap$ $\left[0, t_{2}-t_{2}^{\prime}\right]$.

Acknowledgement. I thank Undine Leopold for careful reading of the manuscript and for valuable suggestions which helped me to improve the presentation.

\section{References}

[AMS1] J. Alonso, H. Martini, M. Spirova, Minimal enclosing discs, circumcircles, and circumcenters on normed planes (Part I), Comput. Geom. 45, 2012, 258-274.

[AMS2] J. Alonso, H. Martini, M. Spirova, Minimal enclosing discs, circumcircles, and circumcenters on normed planes (Part II), Comput. Geom. 45, 2012, 350-369.

[JS] T. Jahn, M. Spirova, On bisectors in normed spaces, Contrib. Discrete Math. 10(2), 2015, 1-9.

[KN] H. Kramer, A.B. Németh, The application of Brouwer's fixed point theorem of convex bodies (Romanian), An. Univ. Timisoara Ser. Sti. Mat. 13, 1977, 33-39. 
[MSW] H. Martini, K.J. Swanepoel, G. Weiss, The geometry of Minkowski spaces - a survey, Part I, Expo. Math. 19, 2001, 97-142.

[MSwa] H. Martini, K.J. Swanepoel, The geometry of Minkowski spaces - a survey, Part II, Expo. Math. 22, 2004, 93-144.

[Ma] A.E. Mayer, Eine Überkonvexität, Math. Z. 39, 1935, 511-531.

[Ne] M.H.A. Newman, Elements of the topology of plane sets of points, Cambridge University Press, 1961.

[Th] A.C. Thompson, Minkowski geometry, Cambridge University Press, 1996.

Matematiikan laitos

Helsingin yliopisto

PL 68

00014 Helsinki, Finland

jussi.vaisala@helsinki.fi 